\title{
The Role of Magnetic Resonance Spectroscopy Imaging Parameters to Predict Early Biochemical Recurrence after Radical Prostatectomy
}

Mehdi Kardoust Parizi ${ }^{1}$, Ali Razi ${ }^{1}$, Soheil Alizadeh ${ }^{1}$, Amir Kasaeian ${ }^{2}$

${ }^{1}$ Department of Urology, Tehran University of Medical Sciences, Shariati Hospital, Tehran, Iran;

${ }^{2}$ Department of Biostatistics and Epidemiology, Tehran University of Medical Sciences, Shariati Hospital, Tehran, Iran

Received December 9, 2018; Accepted September 4, 2019.

Key words: Prostatectomy - Recurrence - MR spectroscopy - Prostate cancer

Abstract: To evaluate the role of magnetic resonance spectroscopy imaging (MRSI) parameters to predict early biochemical recurrence (BCR) after radical prostatectomy (RP) in patients with non-metastatic prostate cancer ( $\mathrm{PCa}$ ).

Between November 2010 and March 2012, 60 consecutive patients with clinically non-metastatic biopsy confirmed PCa underwent RP after MRSI assessment in a prospective study. Demographic, clinicopathological, magnetic resonance imaging (MRI) staging, MRSI parameters, and postoperative serum prostate-specific antigen were recorded. The univariate and multivariate Cox regression analyses were used to assess the association between potential prognosticators and early BCR (BCR less than 12 months after RP). In univariate Cox regression, preoperative serum PSA (prostate-specific antigen) $(H R$ - hazard ratio $=1.016, p=0.003)$, surgical Gleason score $>7(H R=5.034, p=0.006)$ and $M R S I$ risk score $(H R=4.061, p=0.0001)$; and in multivariate model, preoperative serum PSA ( $H R=1.012 ; p=0.046)$, surgical $G S>7(H R=4.196 ; p=0.017)$ and $M R S I$ risk score $(H R=3.256 ; p=0.013)$ were associated with early BCR. The greatest AUC (area under the curve) was related to $M R S I$ risk score $(A \cup C=0.733$ ) and the $A \cup C$ of the multivariate model was 0.776 . $\mathrm{MRI/MRSI}$ parameters specially MRSI risk score might be acceptable predictors of early BCR. These parameters can improve the accuracy of predictive nomograms to assess the risk of BCR after RP.

Mailing Address: Soheil Alizadeh, MD., Shariati Hospital, North Karegar Street, Tehran, Iran; Phone: +982 184901 000; e-mail: s.alizadeh17@gmail.com 


\section{Introduction}

Prostate cancer (PCa) is the second most common cancer in men (Parkin et al., 2005), that consists of $15 \%$ of all new cancers in men (Ferlay et al., 2015). Most of the prognosticators that predict oncological outcomes after radical prostatectomy (RP) are related to the surgical specimen (Kattan et al., 1998; Han et al., 2003; Stephenson et al., 2006). Therefore, pre-operative predictive factors could be valuable for prediction of RP outcomes and also help urologists and patients to perform appropriate decision-making.

Recently, it has been demonstrated that magnetic resonance imaging (MRI) and magnetic resonance spectroscopy imaging (MRSI) are useful to predict oncological outcomes after RP (D'Amico et al., 2000; Pucar et al., 2004; Shukla-Dave et al., 2007).

In this study, we assess MRSI and MRI related prognosticators to predict the early biochemical recurrence $(B C R)$ and time to early $B C R$ after RP in patients with non-metastatic PCa.

\section{Material and Methods}

This study was conducted in agreement with the Declaration of Helsinki and it was approved by the local Ethics Committee. Between November 2010 and March 2012, 60 consecutive patients with biopsy-confirmed non-metastatic PCa were included in this prospective study after signing informed consent. Patients underwent MRI and MRSI six weeks after prostate biopsy. All cases have been subjected to standard open radical retropubic prostatectomy by one surgeon. The mean duration between $\mathrm{MRI} / \mathrm{MRSI}$ and surgery was 29 days. Patients were followed-up by prostate-specific antigen (PSA) 45 days after surgery and every 3 months for the first year and then every 6 months. BCR has been defined as a postoperative PSA $\geq 0.4 \mathrm{ng} / \mathrm{ml}$ followed by an increasing PSA. Early BCR was defined as BCR less than one year after RP. All patients with confirmed metastasis on bone scan or chest/abdominal computed tomography scan before RP or patients with a positive surgical margin after RP were excluded from the study. Demographic, clinicopathological and imaging data including patient's age, preoperative serum PSA, biopsy cores number, malignant biopsy cores percentage, biopsy Gleason score (GS), prostate weight, prostate involvement percentage (in the surgical specimen), and surgical GS were recorded. This study was approved by the ethics committee of the Tehran University of Medical Sciences.

\section{MRSI technique and interpretation}

Patients underwent MRI (T1W, T2W and dynamic contrast-enhanced [DCE]) and multivoxel MRSI with endorectal coil using Siemens Magneto Avanto 1.5 T, 18 Channel T-Class magnetic resonance machine prior to surgery. In MRI, lesions with a hypointense signal on $\mathrm{T} 2 \mathrm{~W}$ without a hyperintense signal on T1W that have been enhanced earlier than or contemporaneously with an enhancement of adjacent normal prostatic tissues were defined as malignant and as "index lesion" (Zakian 
et al., 2010; Weinreb et al., 2016). In index lesion of each patient, the ratio of choline plus creatine to citrate $(\mathrm{Ch}+\mathrm{Cr} / \mathrm{Ci})$ was measured in voxels with $0.216 \mathrm{ml}$ volume. In addition, the voxels according to the quantitative ratio were divided into two groups; healthy voxels (ratio $<0.5$ ) and non-healthy voxels (ratio $\geq 0.5)$. Nonhealthy voxels were categorized into three groups; low-grade voxels $(0.5 \leq$ ratio $\leq 0.6)$, intermediate grade voxels $(0.7 \leq$ ratio $\leq 3)$, and high-grade voxels (ratio $>3)$. Equation: $\frac{(1 \times L G)+(2 \times 1 G)+(3 \times H G)}{100}$ was used to calculate "MRSI risk score" for each

patient (LG: low-grade voxels percentage; IG: intermediate grade voxels percentage; HG: high-grade voxels percentage) (Weinreb et al., 2016). For MRI grouping, a three-point staging (as same as pathologic $T$ stage definition) was used, as follows: 1) tumour confined within prostate $(\mathrm{T} 2) ; 2$ ) tumour extends through the prostate capsule (T3); 3) tumour is fixed or invades adjacent structures other than seminal vesicles, such as external sphincter, rectum, bladder, levator muscles, and/or pelvic wall (T4).

The MRI and MRSI of patients were interpreted by a radiologist with 10 years of experience in prostate imaging interpretation. We matched tumour locations on MRSI and location on step-section pathology maps.

\section{Statistical analysis}

Data were analysed by the statistical package for the social sciences (SPSS Inc., Chicago, IL, USA) software version 22. The overall BCR-free survival graph and BCR-free survival graphs by categorical variables were designed. The univariate Cox regression analysis was used to assess the association between potential prognosticators and early BCR. Continuous variables with a hazard ratio (HR) $>1$ and $p<0.05$ in univariate Cox regression analysis and categorical variables with $p<0.05$ in the log-rank test were considered for a multivariate Cox regression model. AUCs (AUC - area under the curve) were calculated and the ROC (receiver operating characteristic) curve of the multivariate model was plotted. A p-value less than 0.05 was considered statistically significant.

\section{Results}

Demographic, clinical and pathological characteristics of patients are demonstrated in Table 1. The mean patients' age and follow-up duration were 64.2 years (range 46-78 years) and 32.3 months (range 12-90 months), respectively. Nineteen patients (31.7\%) had BCR during 12 months after RP, with the mean time to early BCR of 5.9 months (range 1.5-12 months). BCR-free survival at $3^{\text {rd }}, 6^{\text {th }}$ and $12^{\text {th }}$ month were $91.67 \%$ (81.13-96.44\%), $81.65 \%$ (69.32-89.39\%) and $69.68 \%$ (56.27-79.70\%), respectively.

The mean of MRSI risk scores was 0.91 (range $0-2.37$ ). The mean of $\mathrm{Ch}+\mathrm{Cr} / \mathrm{Ci}$ means of non-healthy voxels was 1.93 (range $0.58-8.44$ ). The mean of maximum $\mathrm{Ch}+\mathrm{Cr} / \mathrm{Ci}$ was 4.83 (range 0.46-19.80). The mean of high-grade voxels 


\section{Table 1 - Demographic, clinical and pathological characteristics of non-metastatic prostate cancer patients treated with radical prostatectomy}

\begin{tabular}{lcc}
\hline Characteristic & Value \\
\hline Age, mean \pm SD (range), years & $64.2 \pm 7.6(46-78)$ \\
\hline Preoperative serum PSA, mean \pm SD (range), ng/ml & $15.2 \pm 24.1(2.3-160)$ \\
\hline Biopsy cores number, mean \pm SD (range) & & $11.3 \pm 5.2(6-27)$ \\
\hline Malignant biopsy cores percentage, mean \pm SD (range) & & $39.0 \pm 17.8(7.5-100)$ \\
\hline Biopsy Gleason score, No. of patients (\%) & $<7$ & $20(33.3)$ \\
& 7 & $30(50.0)$ \\
& $>7$ & $10(16.7)$ \\
\hline Prostate weight, mean \pm SD (range), g & & $50.6 \pm 17.3(13-121)$ \\
\hline Prostate involvement percentage, mean \pm SD (range) & & $22.2 \pm 15.4(1.5-90)$ \\
\hline Surgical Gleason score, No. of patients (\%) & $<7$ & $29(48.3)$ \\
& 7 & $22(36.7)$ \\
\hline
\end{tabular}

PSA - prostate-specific antigen; SD - standard deviation

Graph A: Overall BCR-free survival

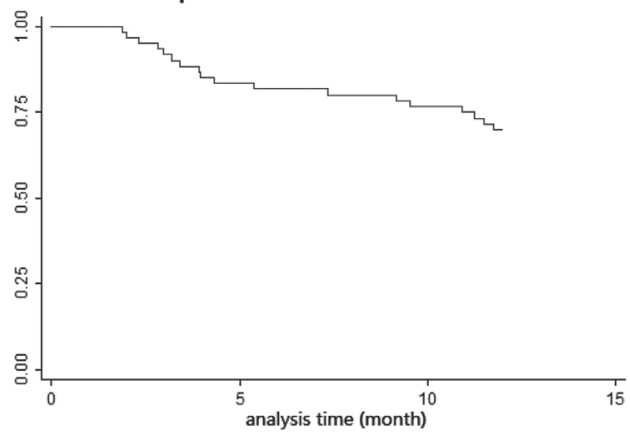

Graph C: BCR-free survival by surgical Gleason score

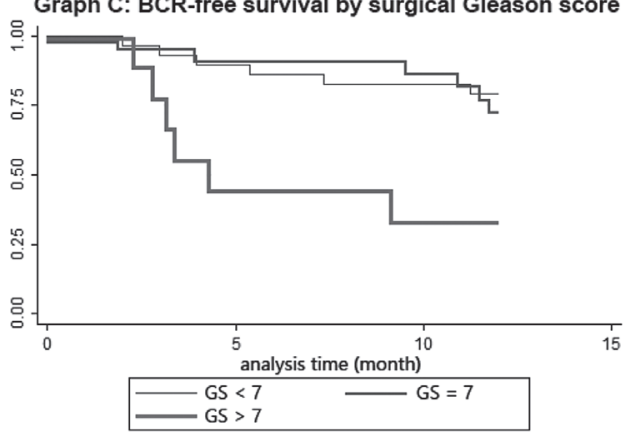

Graph B: BCR-free survival by Pathological T stage

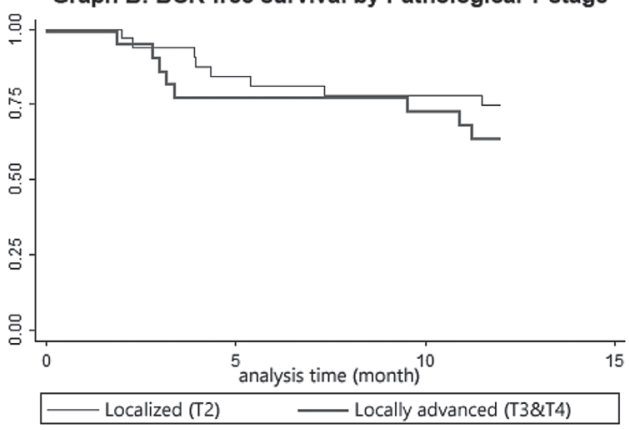

Graph D: BCR-free survival by MRI staging

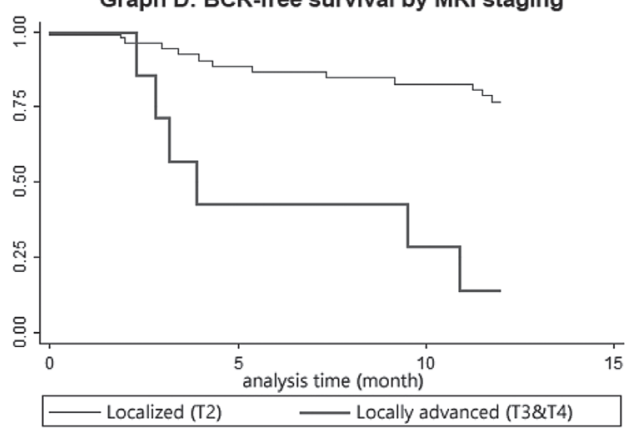

Figure 1 - Kaplan-Meier graphs present overall biochemical recurrence (BCR) free survival and BCR-free survival by three categorical variables.

MRS Related Prognosticators for Biochemical Recurrence after Prostatectomy 


\section{Table 2 - BCR-free survival by categorical prognosticators of prostate cancer patients treated with radical prostatectomy}

\begin{tabular}{|c|c|c|c|c|c|}
\hline & $\begin{array}{l}3^{\text {rd }} \text { month } \\
(95 \% \mathrm{Cl})\end{array}$ & $\begin{array}{l}6^{\text {th }} \text { month } \\
(95 \% \mathrm{Cl})\end{array}$ & $\begin{array}{l}9^{\text {th }} \text { month } \\
(95 \% \mathrm{Cl})\end{array}$ & $\begin{array}{l}12^{\text {th }} \text { month } \\
(95 \% \mathrm{Cl})\end{array}$ & $\begin{array}{c}\text { Log-rank } \\
\text { test } \\
\text { (P-value) }\end{array}$ \\
\hline \multicolumn{6}{|c|}{ BCR-free survival by surgical Gleason score } \\
\hline$<7$ & $\begin{array}{c}93.10 \\
(75.14-98.23)\end{array}$ & $\begin{array}{c}86.14 \\
(67.16-94.56)\end{array}$ & $\begin{array}{c}82.55 \\
(63.02-92.34)\end{array}$ & $\begin{array}{c}78.88 \\
(58.87-89.92)\end{array}$ & \\
\hline$=7$ & $\begin{array}{c}95.45 \\
(71.87-99.35)\end{array}$ & $\begin{array}{c}90.91 \\
(68.30-97.65)\end{array}$ & $\begin{array}{c}90.91 \\
(68.30-97.65)\end{array}$ & $\begin{array}{c}72.73 \\
(49.10-86.71)\end{array}$ & 0.004 \\
\hline$>7$ & $\begin{array}{c}77.78 \\
(36.48-93.93) \\
\end{array}$ & $\begin{array}{c}44.44 \\
(13.59-71.93) \\
\end{array}$ & $\begin{array}{c}33.33 \\
(7.83-62.26)\end{array}$ & $\begin{array}{c}33.33 \\
(7.83-62.26)\end{array}$ & \\
\hline \multicolumn{6}{|c|}{ BCR-free survival by pathological $T$ stage } \\
\hline $\begin{array}{l}\text { Localized } \\
\text { (T2) } \\
\text { Locally advanced } \\
\text { (T3 and T4) }\end{array}$ & $\begin{array}{c}93.75 \\
(77.25-98.40) \\
86.36 \\
(63.44-95.39)\end{array}$ & $\begin{array}{c}81.19 \\
(62.84-91.08) \\
77.27 \\
(53.74-89.85)\end{array}$ & $\begin{array}{c}77.94 \\
(59.21-88.83) \\
77.27 \\
(53.74-89.85)\end{array}$ & $\begin{array}{c}74.63 \\
(55.57-86.43) \\
63.64 \\
(40.29-79.88)\end{array}$ & 0.369 \\
\hline \multicolumn{6}{|c|}{ BCR-free survival by MRI staging } \\
\hline $\begin{array}{l}\text { Localized } \\
\text { (T2) } \\
\text { Locally advanced } \\
\text { (T3 and T4) }\end{array}$ & $\begin{array}{c}94.23 \\
(83.17-98.10) \\
71.43 \\
(25.82-91.98)\end{array}$ & $\begin{array}{c}86.52 \\
(73.79-93.33) \\
42.86 \\
(9.78-73.44)\end{array}$ & $\begin{array}{c}84.55 \\
(71.47-91.96) \\
42.86 \\
(9.78-73.44)\end{array}$ & $\begin{array}{c}76.61 \\
(62.49-85.99) \\
14.29 \\
(0.71-46.49)\end{array}$ & 0.0001 \\
\hline
\end{tabular}

$\mathrm{BCR}$ - biochemical recurrence; $\mathrm{MRI}$ - magnetic resonance imaging; $\mathrm{Cl}$ - confidence interval

percentage was $14.6 \%$ (range $0-50 \%$ ). The mean of non-healthy voxels percentage and number were $45.6 \%$ and 6.1 , respectively.

The overall BCR-free survival graph and BCR-free survival graphs by categorical variables are shown in Figure 1. There was a statistically significant difference between the surgical GS groups $(p=0.003)$ and MRI staging groups $(p=0.0001)$, in the log-rank test (Table 2).

Table 3 presents associations between MRI/MRSI parameters and early BCR in univariate and multivariate analyses and AUCs. Preoperative serum PSA $(H R=1.016, p=0.003)$, surgical $G S>7(H R=5.034, p=0.006)$ and MRSI risk score $(H R=4.061, p=0.0001)$ were associated with early $B C R$ in univariate Cox regression analysis. In the multivariate Cox regression model, preoperative serum PSA ( $H R=1.012 ; p=0.046)$, surgical $G S>7(H R=4.196 ; p=0.017)$ and MRSI risk score $(H R=3.256 ; p=0.013)$ were associated with early $B C R$. Figure 2 illustrates the ROC curve of the multivariate model predicting early BCR. The greatest AUC was related to MRSI risk score (AUC = 0.733). The AUC for the multivariate model was 0.776 , which confirms a greater predictive value of multivariate model than each variable. Figure 3 shows a sample of index lesion consisted of 3 voxels with high $\mathrm{Ch}+\mathrm{Cr} / \mathrm{Ci}$ ratio. 


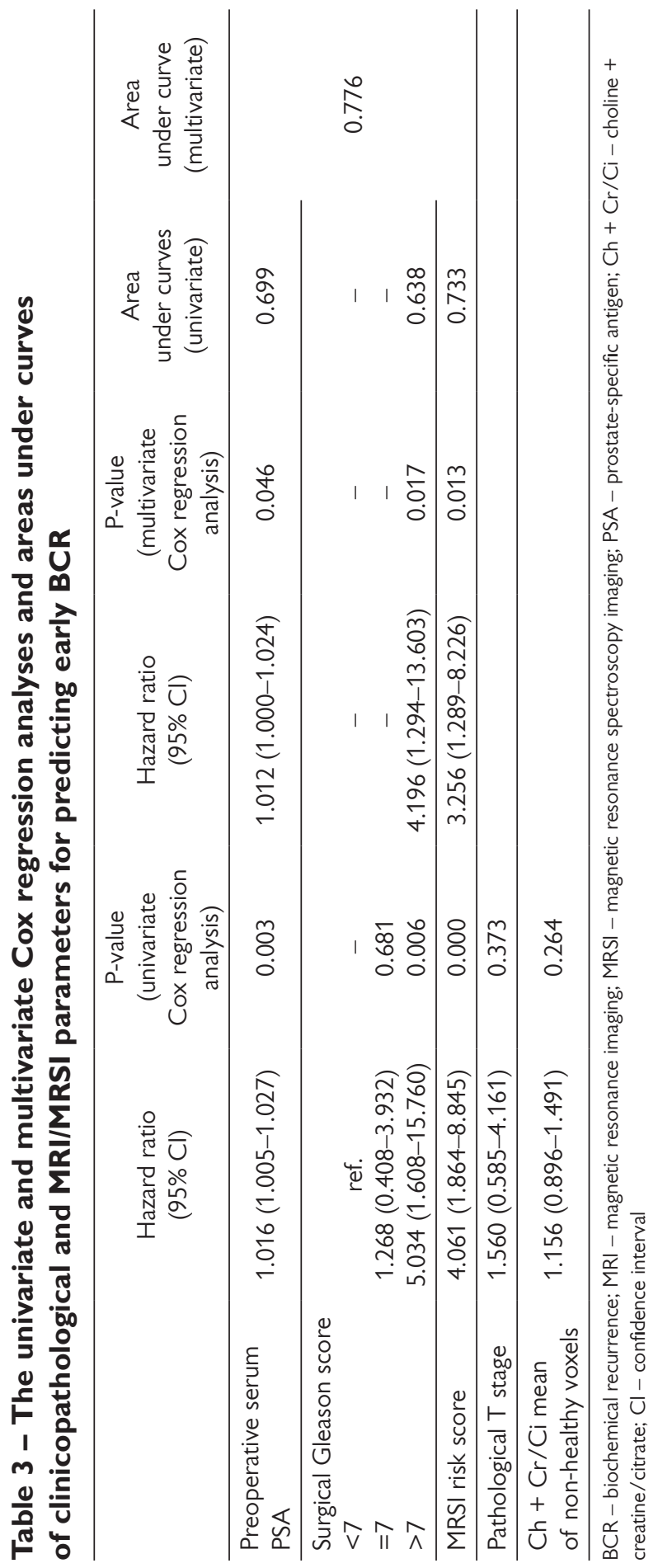




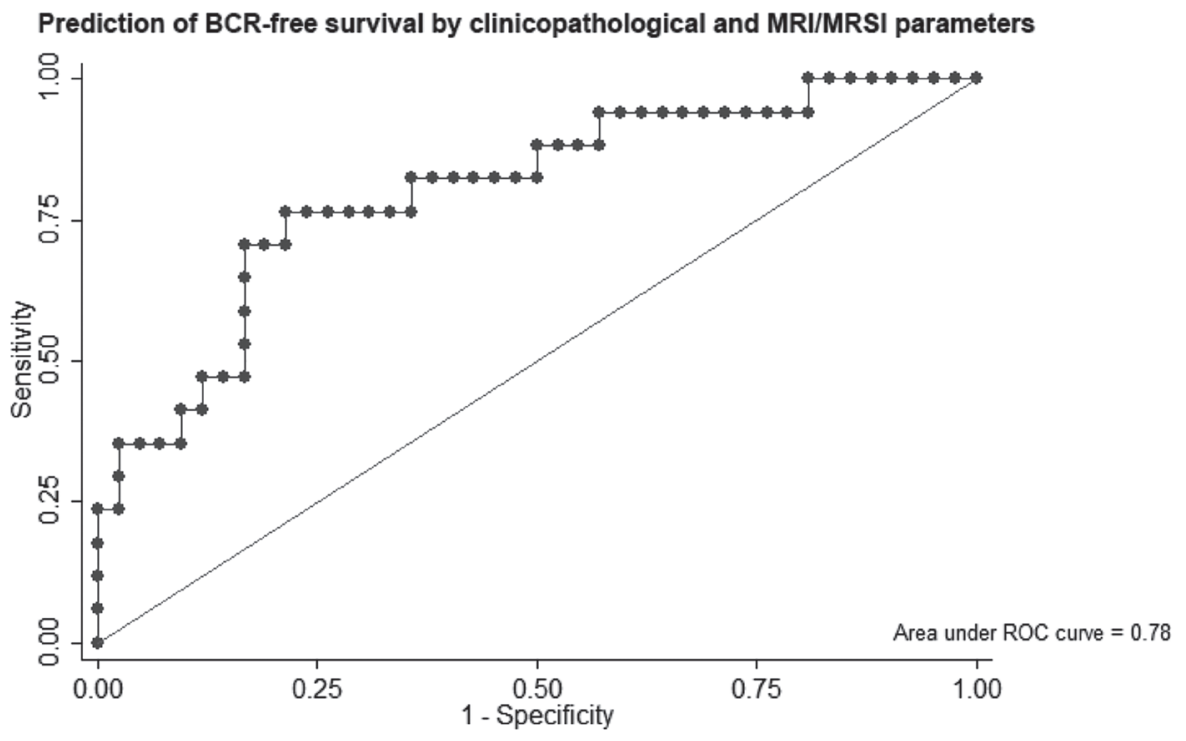

Figure $2-R O C$ (receiver operating characteristic) curve of clinicopathological and MRI/MRSI parameters (preoperative serum PSA, surgical Gleason score and MRSI - magnetic resonance spectroscopy imaging risk score) in the prediction of biochemical recurrence $(B C R)$ free survival in a multivariate analysis.
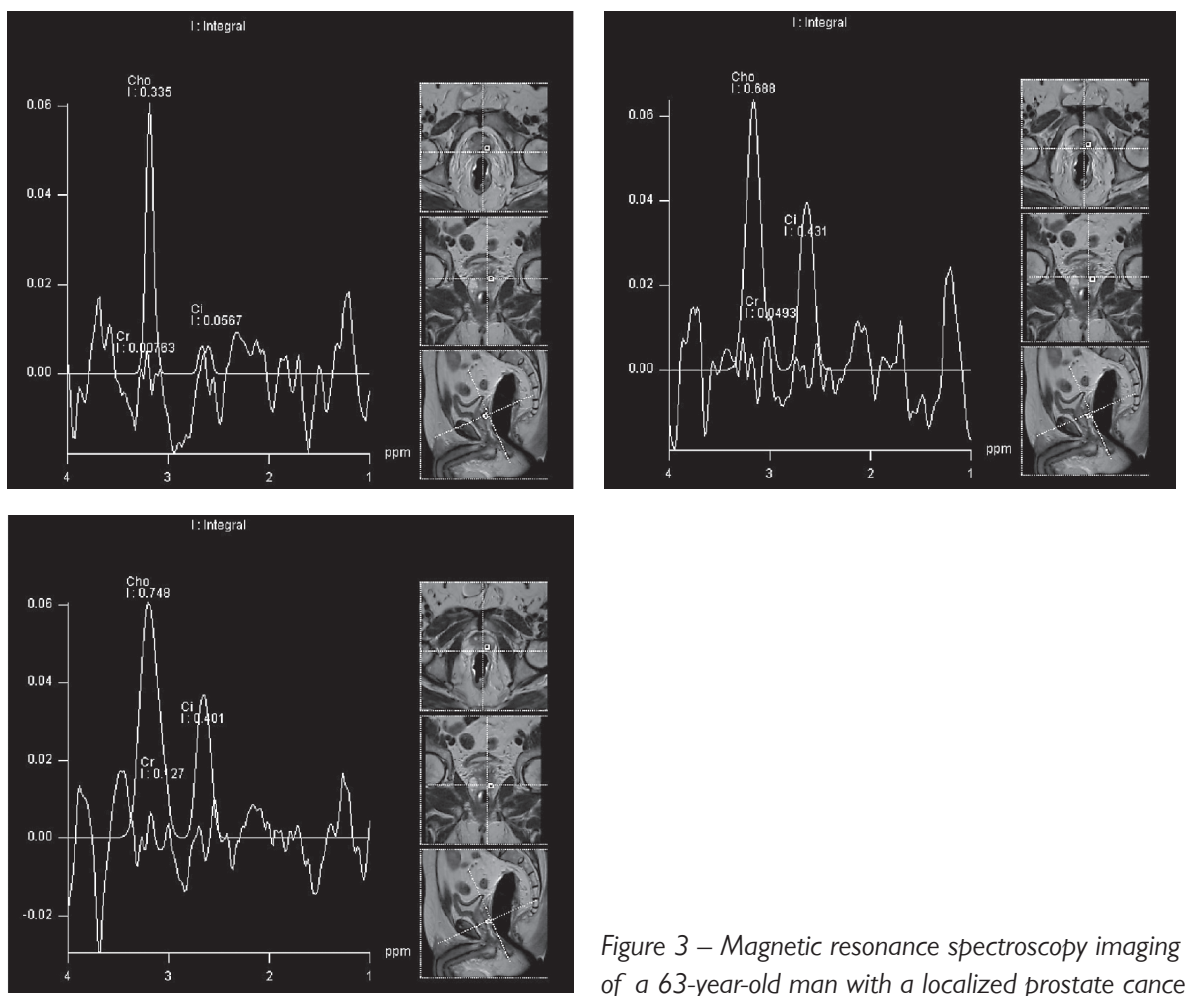

Figure 3 - Magnetic resonance spectroscopy imaging of a 63-year-old man with a localized prostate cancer. 


\section{Discussion}

BCR after RP represents a local or distant cancer control failure and has a critical role in the patient's management. The incidence of post-RP BCR is estimated up to 45\% (Loeb et al., 2011; Liesenfeld et al., 2017). Both early and late BCR are related to PCa specific death (Ferlay et al., 2015). Pound et al. (1999) showed that time to BCR was significantly associated with the development of metastatic disease in patients with PCa. Therefore, accurate prognostic models predicting early disease recurrence might help to perform a proper decision-making process such as neoadjuvant treatments.

Several parameters including patient's age, preoperative PSA, surgical GS, and pathological stage, are frequently used variables to predict biochemical failure after RP (Liesenfeld et al., 2017). Some validated nomograms have been designed to predict BCR and relapse after definitive treatment of PCa (Kattan et al., 1998; Han et al., 2003; Stephenson et al., 2006).

Stephenson et al. (2006) evaluated the long-term post RP recurrence in a predictive nomogram including clinical stage, serum PSA, and biopsy Gleason grade, number of positive and negative biopsy cores, and year of surgery. The authors concluded that these parameters might improve the accuracy of the previously explained nomogram to predict 1 to 10 years' progression-free survival after RP.

Zakian et al. (2010), assessed the prognosticative value of MRI/MRSI findings to predict BCR after RP in 132 patients with PCa. They presented the MRSI index lesion volume and the presence of high-grade MRSI voxels as valuable parameters to predict time to BCR after RP. These new modalities can be used to improve the accuracy of oncological outcomes predictor nomograms (Zhang et al., 2017).

In this study, we evaluated several MRI/MRSI parameters in details to find a reasonable correlation between these prognosticators and early BCR. KaplanMeier survival graphs for biochemical recurrence-free survival demonstrated a significant association between surgical GS and MRI staging and early BCR. MRSI risk score has a greater AUC (0.733) in comparison with other predictive factors. Moreover, surgical Gleason score $>7$ was a significant factor to predict early BCR in the multivariate analysis. The statistical value of MRSI parameters to predict the oncological outcome after RP has been analysed by some investigators. Nomograms including MRI/MRSI parameters might be more accurate to predict oncological outcomes of patients after RP in comparison with popular nomograms such as D'Amico and cancer of the prostate risk assessment (CAPRA) score (Zhang et al., 2015, 2017).

Zhang et al. (2017) in an MRI-based analysis of predictors assessed the oncologic outcomes after RP in 205 patients with PCa in a retrospective study. They compared the performance of MRI-based nomogram with D'Amico and CAPRA schemes in term of 3 years BCR after RP and found a greater AUC for MRI-based nomogram (AUC 0.909 for MRI-based nomogram versus 0.901 and 0.894 for D'Amico and CAPRA, respectively). 
We excluded patients with a positive surgical margin to modify cofounder effect of such an important predictor affecting early BCR. No patient received any neoadjuvant or adjuvant therapy before BCR. So, these predictors might be helpful to find cases with clinically organ-confined PCa that may benefit from neoadjuvant or early adjuvant treatments.

The present study has some limitations. First, short-term follow-up of 32.3 months could be considered a limitation of our study. As we analysed early BCR rate (biochemical recurrence less than 12 months), this follow-up period can be regarded sufficient. Second, the small sample size of the present study makes robust recommendation difficult. The results of this study may be confirmed by future prospective large sample studies.

\section{Conclusion}

$\mathrm{MRSI} / \mathrm{MRI}$ parameters might be useful to find high-risk patients for early BCR. These potential prognosticators can influence the surgeon and the patient's decisionmaking process to select an appropriate treatment strategy. Moreover, MRSI/MRI parameters might be included in predictive nomograms to increase the accuracy of these prognostic tools in patients with PCa.

\section{References}

D’Amico, A. V., Whittington, R., Malkowicz, B., Schnall, M., Schultz, D., Cote, K., Tomaszewski, J. E., Wein, A. (2000) Endorectal magnetic resonance imaging as a predictor of biochemical outcome after radical prostatectomy in men with clinically localized prostate cancer. J. Urol. 164(3), 759-763.

Ferlay, J., Soerjomataram, I., Dikshit, R., Eser, S., Mathers, C., Rebelo, M., Parkin, D. M., Forman, D., Bray, F. (2015) Cancer incidence and mortality worldwide: Sources, methods and major patterns in GLOBOCAN 2012. Int. J. Cancer 136(5), E359-E386.

Han, M., Partin, A. W., Zahurak, M., Piantadosi, S., Epstein, J. I., Walsh, P. C. (2003) Biochemical (prostate specific antigen) recurrence probability following radical prostatectomy for clinically localized prostate cancer. J. Urol. 169(2), 517-523.

Kattan, M. W., Eastham, J. A., Stapleton, A. M., Wheeler, T. M., Scardino, P. T. (1998) A preoperative nomogram for disease recurrence following radical prostatectomy for prostate cancer. J. Natl. Cancer Inst. 90(10), 766-771.

Liesenfeld, L., Kron, M., Gschwend, J. E., Herkommer, K. (2017) Prognostic factors for biochemical recurrence more than 10 years after radical prostatectomy. J. Urol. 197(1), 143-148.

Loeb, S., Feng, Z., Ross, A., Trock, B. J., Humphreys, E. B., Walsh, P. C. (2011) Can we stop prostate specific antigen testing 10 years after radical prostatectomy? J. Urol. 186(2), 500-505.

Parkin, D. M., Bray, F., Ferlay, J., Pisani, P. (2005) Global cancer statistics, 2002. CA Cancer J. Clin. 55(2), 74-108.

Pound, C. R., Partin, A. W., Eisenberger, M. A., Chan, D. W., Pearson, J. D., Walsh, P. C. (1999) Natural history of progression after PSA elevation following radical prostatectomy. JAMA 281(17), 1591-1597.

Pucar, D., Koutcher, J. A., Shah, A., Dyke, J. P., Schwartz, L., Thaler, H., Kurhanewicz, J., Scardino, P. T., Kelly, W. K., Hricak, H., Zakian, K. L. (2004) Preliminary assessment of magnetic resonance spectroscopic imaging in predicting treatment outcome in patients with prostate cancer at high risk for relapse. Clin. Prostate Cancer 3(3), 174-181. 
Shukla-Dave, A., Hricak, H., Kattan, M. W., Pucar, D., Kuroiwa, K., Chen, H. N., Spector, J., Koutcher, J. A., Zakian, K. L., Scardino, P. T. (2007) The utility of magnetic resonance imaging and spectroscopy for predicting insignificant prostate cancer: an initial analysis. BJU Int. 99(4), 786-793.

Stephenson, A. J., Scardino, P. T., Eastham, J. A., Bianco, F. J. Jr., Dotan, Z. A., Fearn, P. A., Kattan, M. W. (2006) Preoperative nomogram predicting the 10-year probability of prostate cancer recurrence after radical prostatectomy. J. Natl. Cancer Inst. 98(10), 715-717.

Weinreb, J. C., Barentsz, J. O., Choyke, P. L., Cornud, F., Haider, M. A., Macura, K. J., Margolis, D., Schnall, M. D., Shtern, F., Tempany, C. M., Thoeny, H. C. (2016) PI-RADS Prostate Imaging - Reporting and Data System: 2015, Version 2. Eur. Urol. 69(1), 16-40.

Zakian, K. L., Hricak, H., Ishill, N., Reuter, V. E., Eberhardt, S., Moskowitz, C. S., Shukla-Dave, A., Wang, L., Scardino, P. T., Eastham, J. A., Koutcher, J. A. (2010) An exploratory study of endorectal MRI and spectroscopy of the prostate as pre-operative predictive biomarkers of biochemical relapse after radical prostatectomy. J. Urol. 184(6), 2320.

Zhang, Y. D., Wang, Q., Wu, C. J., Wang, X. N., Zhang, J., Liu, H., Liu, X. S., Shi, H. B. (2015) The histogram analysis of diffusion-weighted intravoxel incoherent motion (IVIM) imaging for differentiating the Gleason grade of prostate cancer. Eur. Radiol. 25(4), 994-1004.

Zhang, Y. D., Wu, C. J., Bao, M. L., Li, H., Wang, X. N., Liu, X. S., Shi, H. B. (2017) MR-based prognostic nomogram for prostate cancer after radical prostatectomy. J. Magn. Reson. Imaging 45(2), 586-596. 